\title{
High-density mapping of quantitative trait loci for grain-weight and spikelet number in rice
}

\author{
Dong-Min Kim 1,4, Hyun-Sook Lee', Soo-Jin Kwon², Mark Edward Fabreag ${ }^{1}$, Ju-Won Kang ${ }^{1}$, Yeo-Tae Yun ${ }^{3}$, \\ Chong-Tae Chung ${ }^{3}$ and Sang-Nag Ahn ${ }^{1 *}$
}

\begin{abstract}
Background: High grain yield is one of the most important traits requiring improvement in rice breeding programs. Consequently, the genetic basis of spikelets per panicle (SPP) and grain weight (TGW) have received much research focus because of their importance in rice yield.

Results: In this study, IL28, which is a near isogenic line (NIL) developed by introgressing chromosomal segments of the cultivar 'Moroberekan' into the cultivar 'Ilpumbyeo', showed a significant increase in the number of spikelets per panicle (SPP) and 1,000-grain weight (TGW) compared to the recurrent parent, Ilpumbyeo. Quantitative trait locus (QTL) analysis in 243 F2 plants derived from a cross between IL28 and Ilpumbyeo indicated that both qSPP6 and qTGW6 are located in the interval RM3430-RM20580. Following substitution mapping with 50 F:4:5 lines, qSPP6 was mapped to a 429-kb interval between RM20521 and InDel-1, while qTGW6 was mapped to a 37.85-kb interval between InDel-1 and SNP-3 based on the japonica genome sequence. This result indicates that qSPP6 and qTGW6 are different genes. Yield trials with substitution lines indicated that lines harboring the homozygous Moroberekan segment at both the 9SPP6 and qTGW6 region showed significantly higher grain yield than Ilpumbyeo.
\end{abstract}

Conclusion: Because the Moroberekan alleles for SPP and TGW have been shown to be beneficial in the genetic background of Ilpumbyeo, both the QSPP6 and qTGW6 alleles might prove valuable in improving rice yields. Closely linked SSR markers are expected to facilitate the cloning of genes that underlie these QTLs, as well as with marker-assisted selection for variation in SPP and TGW in rice breeding programs.

Keywords: Rice; Spikelets per panicle; Grain weight; QTL; Linkage; Near isogenic line

\section{Background}

Rice (Oryza sativa L.) is the world's most important cereal food crop. The anticipated rapid increase in the global human population, which is expected to reach 9.1 billion by 2050, might generate serious food shortage problems. Moreover, various factors, such as water scarcity, soil salinity, disease, climate change, and reduced arable land, will exacerbate food shortages in the next 50 years (Khush 1999 and Khush 2005; Zhang 2007). Therefore, researchers are focusing on increasing existing crop grain yield levels. Grain weight, spikelets per panicles, and the number of panicles per plant are the most important components of grain yield. However, the genetic analysis of these three yield components is difficult, because these traits are controlled

\footnotetext{
* Correspondence: ahnsn@cnu.ac.kr

'Department of Agronomy, College of Agriculture \& Life Sciences,

Chungnam National University, Daejeon 305-764, South Korea

Full list of author information is available at the end of the article
}

by multiple genes, in addition to being influenced by the environment. Therefore, the advent of molecular maps for rice (Causse et al. 1994) and quantitative trait locus (QTL) analysis approaches have facilitated the analysis of these quantitative traits.

Genes/QTLs for spikelets per panicle (SPP) have been reported in populations derived from inter-specific crosses (Xiong et al. 1999; Thomson et al. 2003; Suh et al. 2005; Li et al. 2006 and Linh et al. 2008), indica-indica crosses (Lin et al. 1996; Zhuang et al. 1997), and inter-subspecific crosses (Yamagishi et al. 2002; Ando et al. 2008). To date, a few QTLs associated with SPPs have been detected, including Gn1a, APO1, DEP1, and OsSPL14. Gn1a controls grain number in rice, and was found to encode cytokinin oxidase/dehydrogenase (OsCKX2), which is an enzyme that degrades the phytohormone cytokinin (Ashikari et al. 2005). Higher expression of OsSPL14 during the reproductive stage of rice promotes panicle 
branching leading to higher grain yield (Miura et al. 2010). DEP1, which alters panicle architecture, and hence increases grain yield, has also been identified (Huang et al. 2009). Another approach to identify the genes related to panicle architecture is through the analysis of mutants that alter panicle structure. The characterization of the aberrant panicle organization 1 (apo1) mutant in rice revealed that $A P O 1$ positively controls spikelet number by suppressing the precocious conversion of inflorescence meristems to spikelet meristems (Ikeda et al. 2007). Ookawa et al. (2010) also reported that a near isogenic line carrying APO1 enhanced culm strength and increased spikelet number per panicle.

Grain weight $(\mathrm{GW})$ is another characteristic that is targeted to enhance rice yield. Many QTLs for grain size have been identified in rice populations based on crosses between divergent cultivars or accessions (Lin et al. 1996; Cui et al. 2003; Ishimaru 2003; Zhang et al. 2009). Several genes that regulate grain weight have been identified, including GS3, GS5, GW5, GW2, and TGW6. GW2 is a QTL for rice grain width and weight, which encodes a previously unknown RING-type E3 ubiquitin ligase that negatively regulates cell division by targeting its substrate (s) for degradation by the ubiquitin-proteasome pathway (Song et al. 2007). Ishimaru et al. (2013) found that when the IAA-glucose hydrolase gene stops functioning, TGW6 enhances grain weight through pleiotropic effects on source organs, and leads to significant yield increases.

Numerous studies have reported that one genomic region harbors more than two independent genes that are closely linked, or a gene with pleiotropic effect on several traits (Xie et al. 2008; Jin et al. 2009; Lou et al. 2013; Ji et al., 2014). Xie et al. (2008) reported that two QTLs, sn9.1 and gw9.1, for 1,000-grain weight and spikelets per panicle were identified in the 37.4-kb interval flanked by markers RM24718 and RM30005. Two QTLs acted in an additive manner in this region, resulting in the NIL with the O. rufipogon alleles exhibiting significantly higher SPP and TGW values compared to the recurrent parent, Hwaseongbyeo (Xie et al. 2008). However, it remains unclear whether either of the two closely linked QTLs, or one pleiotropic QTL, are associated with the observed variation. A large-sized population combined with high-density mapping is required to determine whether target traits are controlled by closely linked QTLs, or one pleiotropic QTL. In previous studies (Ju et al. 2008), IL28 was developed, which is an introgression line derived from a cross between the cultivars Ilpumbyeo and Moroberekan, showing higher SPP and TGW values compared to Ilpumbyeo. Kim et al. (2013) reported that a QTL for spikelets per panicle was located between RM20521 and RM20572 using $F_{2: 3}$ populations derived from a cross between Ilpumbyeo and IL28. The present study was carried out to detect a novel QTL for TGW, and to clarify its relationship to the $q$ SPP6 QTL using $\mathrm{F}_{3: 4: 5}$ populations.

\section{Results}

\section{Characteristics of IL28}

In our previous study (Kim et al. 2013), the genetic map of IL28 was constructed using 134 markers that exhibited polymorphism between Ilpumbyeo and Moroberekan, with 2 Moroberekan introgression segments being detected on chromosomes 4 and 6 . The phenotypic evaluation of agronomic traits for the introgression line, IL28, and the recurrent parent was conducted at two locations, Daejeon and Yeasan, in 2013. Comparison of 10 agronomic traits between IL28 and Ilpumbyeo obtained in the current study are shown in Table 1 and Figure 1. The results indicated that there were highly significant differences $(P<0.01)$ in panicle length $(\mathrm{PL})$, secondary branch number $(\mathrm{SBN})$, spikelets per panicle (SPP), first node width (FNW), second node width (SNW), and 1,000-grain weight between IL28 and Ilpumbyeo, while no significant difference was obtained for tiller number (TN) and culm length (CL). Because IL28 has a large endosperm and heavier grain, we investigated the grain milk-filling rate in IL28 and Ilpumbyeo. No difference was observed in either endosperm fresh weight or dry weight at $3 \mathrm{~d}$ and $6 \mathrm{~d}$ after fertilization (Figure 2). In comparison, both the fresh and dry weight of IL28 were significantly higher $(P<0.01)$ compared to those of Ilpumbyeo at $11 \mathrm{~d}$ after fertilization. These differences reached a maximum $\sim 25 \mathrm{~d}$ after fertilization, at which point the fresh and dry weight of the endosperm of IL28 were $11.2 \%$ and $13.4 \%$ higher compared to Ilpumbyeo, respectively. Thus, an increase in grain weight in IL28 which is associated with higher grain milk-filling rate might be due to the Moroberekan segment on chromosome 6 considering that no QTL for grain width and weight was detected in the Moreberekan segment on chromosome 4 .

\section{Trait variation of the $F_{2}$ population}

In our previous study (Kim et al. 2013), four traits (panicle length, numbers of primary branches and secondary branches, and spikelets per panicle) exhibited continuous and normal distributions in the $243 \mathrm{~F}_{2}$ plants. The 250grain weight of Ilpumbyeo and IL28 was 5.51 and 6.14, respectively. In all traits measured, transgressive plants with higher or lower mean values compared to either parent were observed. The correlation between spikelets per panicle and grain weight was not significant $(r=0.102)$.

\section{QTL analysis}

Three significant QTLs for panicle length, secondary branch number, and spikelets per panicle were detected. These QTLs occurred in the same region, near the SSR 
Table 1 Comparison of 10 agronomic traits between IL28 and Ilpumbyeo at two locations in 2013 Traits Mean \pm S.D.

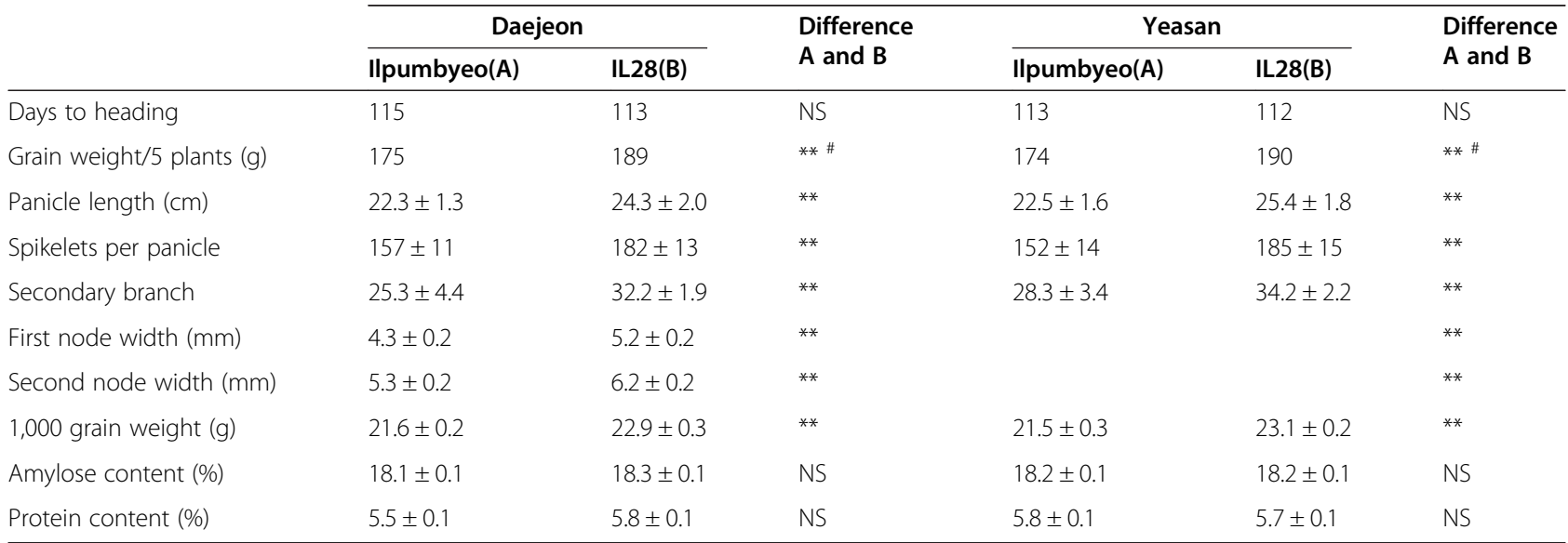

Data were from the trial 2013 in Daejeon and Yeasan. Comparison of two lines was carried out at Daejeon for three years from 2011 to 2013, and the results were nearly the same. Only the 2013 data from Daejeon are presented.

-Not evalutated

\#**Significant at $P<0.01, \mathrm{NS}$ : not significant.

marker RM3430 (Kim et al. 2013). In addition, the QTL for grain weight was detected in the same region, on chromosome 6. The QTL for grain weight explained $8.3 \%$ of phenotypic variance. The Moroberekan alleles increased all trait values of panicle length, secondary branch number, spikelets per panicle and grain weight at this region.

\section{Substitution mapping of qTGW6}

To confirm and narrow down the target region containing $q$ TGW6, 1,120 $\mathrm{F}_{2}$ plants derived from a cross between Ilpumbyeo and IL28 were genotyped with 4 additional markers near the target region (RM20512, RM20521, RM20562, and RM20572) and one marker (RM551) located on chromosome 4. A total of $44 F_{2}$ homozygous plants with different cross-over breakpoints between RM7269 and RM20653 on chromosome 6 were selected and selfed to produce $F_{3: 4: 5}$ lines. Genotype analysis indicated that these $44 \mathrm{~F}_{2}$ plants did not have Moroberekan segment on chromosome 4. According to the marker genotype, the $44 \mathrm{~F}_{3: 4: 5}$ lines were divided into 11 groups, and used for the substitution mapping of $q T G W 6$ (Figure 3). The mean phenotypic values of each trait for each $F_{3: 4: 5}$ line was compared to those of Ilpumbyeo and IL28 at the $P<0.05$ level. In a previous study, the QTLs for SPP, PL, SBM, FNW, and SNW were consistently mapped between RM20521 and RM20572, which are 680-kb apart (Kim et al. 2013). For the $q T G W 6$ locus, groups D, E, F, G, H, I, and J showed significantly higher values than Ilpumbyeo for 1,000-grain (a)

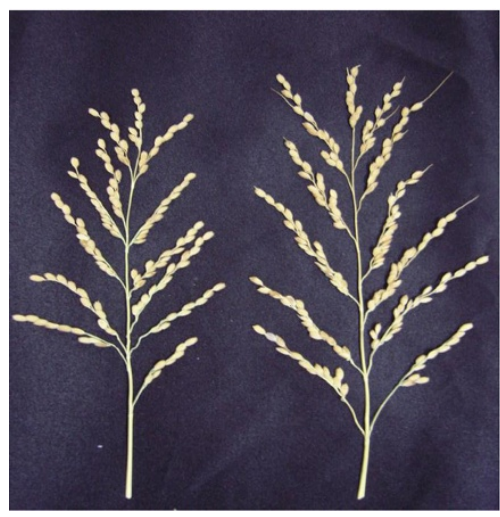

Ilpumbyeo
IL28 (b)

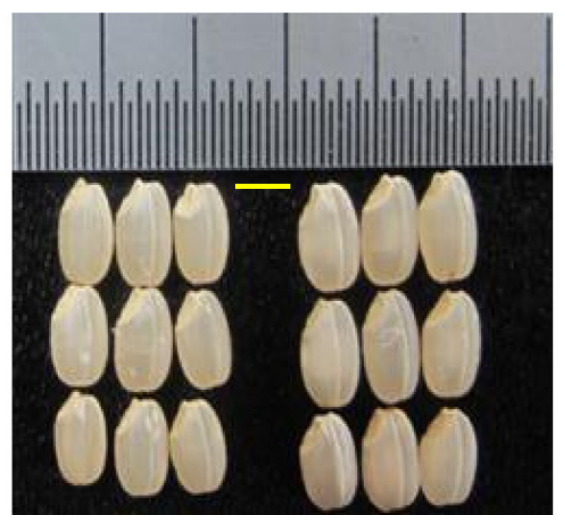

Ilpumbyeo
IL28 (c)

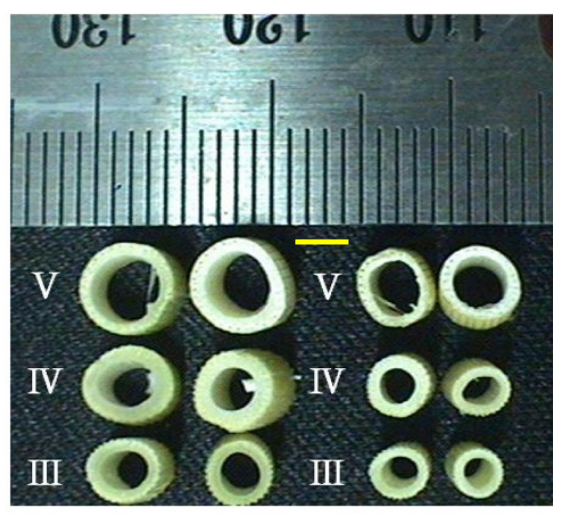

IL28

Ilpumbyeo

Figure 1 Phenotype difference between Ilpumbyeo and IL28 in (a) panicle, (b) grain size (scale bar: 3 mm), and (c) node width (scale bar: $3 \mathrm{~mm}$ ). III, IV, and $V$ indicate the third, fourth, and fifth nodes, respectively. 

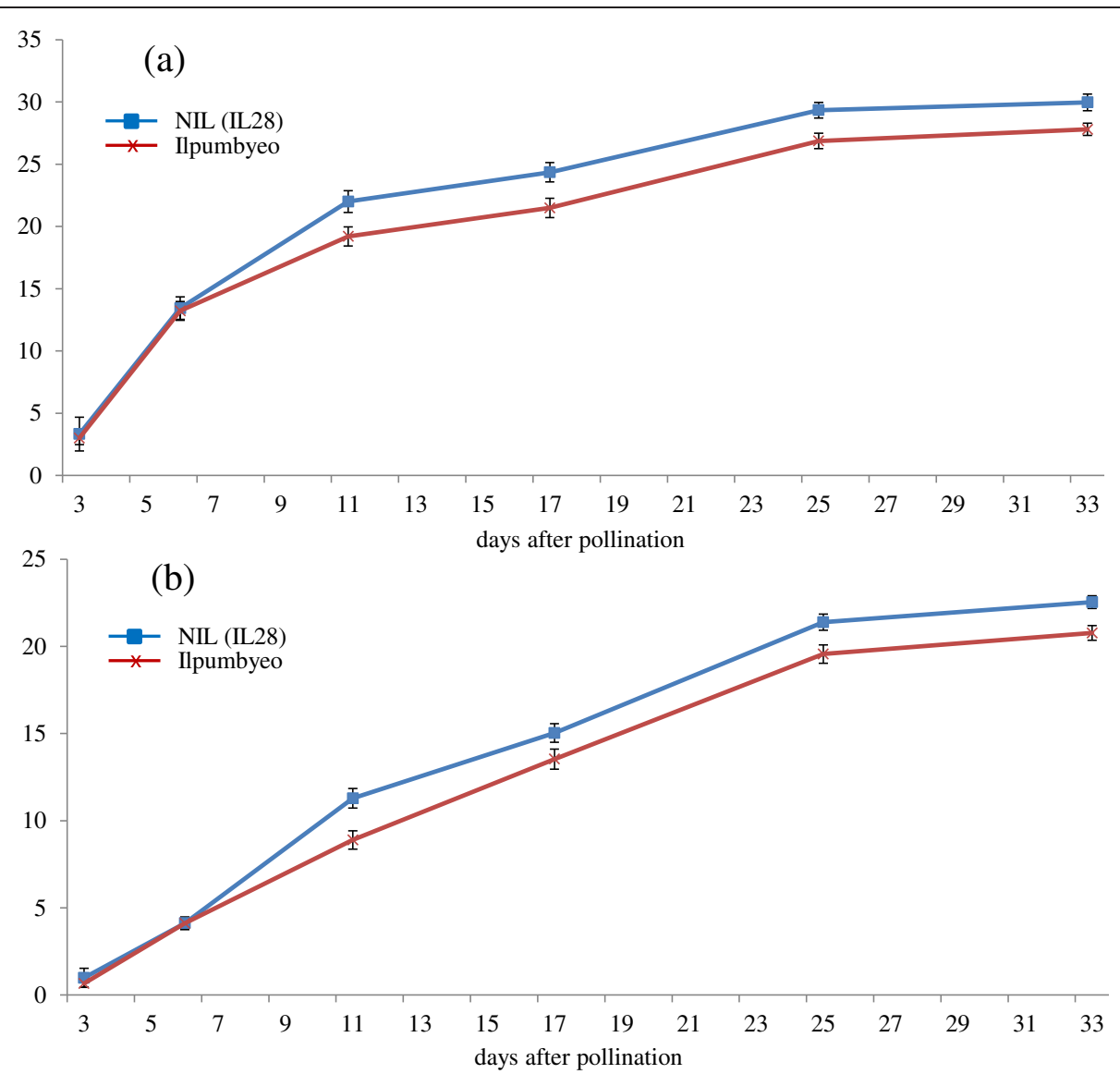

Figure $\mathbf{2}$ Characterization of grain filling in Ilpumbyeo and IL28. (a) Time-course of endosperm fresh weight increase. (b) Time-course of endosperm dry weight increase. Data are mean \pm s.d. ( $n=7$ to 10 plants) in $\mathbf{a}$ and $\mathbf{b}$.

weight, whereas no significance difference was detected between Ilpumbyeo and groups A, B, C, and K in 2011, 2012, and 2013 (Figure 3a). Thus, qTGW6 was located downstream of RM20521 and upstream of RM20580. Group D showed significantly higher values for SPP affected by $q S P P 6$ and for TGW compared to Ilpumbyeo, while group J was significantly different from Ilpumbyeo for TGW but not SPP. This result indicates that qSPP6 and qTGW6 are different genes. Comparison of the genotypes of recombinants delimited the qTGW6 between markers RM20562 and RM20572, based on the finding that the TGW of both D and J groups were significantly different from that of Ilpumbyeo. The region between the two markers RM20562 and RM29572 for groups D and J shared common segment harboring of $q T G W 6$. To further confirm the common segment shared by groups $\mathrm{D}$ and $\mathrm{J}$, we genotyped the $\mathrm{F}_{3}$ lines using four markers (one InDel marker and three SNP markers) located between RM20562 and RM20572. The genotypes showed that qTGW6 was defined in a $37.85-\mathrm{kb}$ region between InDel-1 and SNP3 (Figure 3b).

\section{Evaluation of the grain shape trait}

To determine which grain shape traits are associated with increase in grain weight, three grain shape traits (specifically, grain length $[\mathrm{GL}]$, width $[\mathrm{GW}]$, and thickness $[\mathrm{GT}]$ ) were evaluated for the two parents and $\mathrm{F}_{5}$ lines (Figure 3). The mean phenotypic values of each $F_{5}$ line were compared to those of Ilpumbyeo and IL28 at the $P<0.05$ level. The GL of Ilpumbyeo and IL28 were $4.88 \mathrm{~mm}$ and $5.13 \mathrm{~mm}$, respectively. The GW values for Ilpumbyeo and IL28 were $2.93 \mathrm{~mm}$ and $3.15 \mathrm{~mm}$, respectively. The GL and GW values of IL28 were significantly higher compared to Ilpumbyeo $(P<0.05)$, whereas no significant difference was detected for GT. The GL and GW of the groups with qTGW6 (D, E, F, G, H, I, and $J$ ) were significantly different from those of the groups without $q T G W 6$ (A, B, C, and $\mathrm{K})$. This finding indicates that $q T G W 6$ variation is caused by variation in GL and GW at qTGW6. Grain weight was significantly correlated with GL and GW $(P<0.01$ for both characteristics), but not with GT $(r=-0.06)$. 


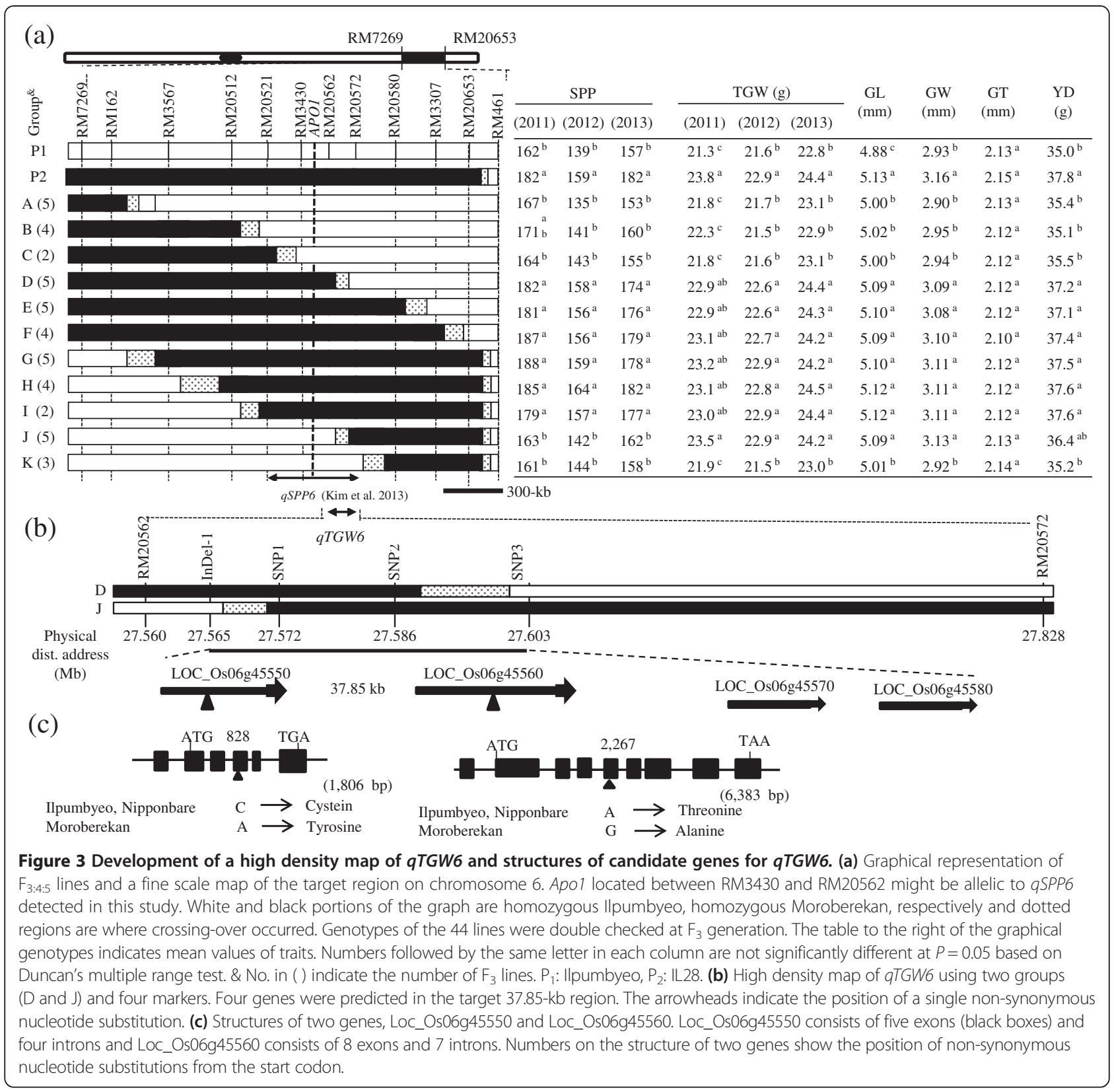

Cross-section of the central part of the spikelet hull

Given that the hull of the IL28 spikelet was wider than that of Ilpumbyeo before fertilization, the cross-section of the central part of the spikelet hull for groups D and J was analyzed in comparison to Ilpumbyeo to investigate the origins of the observed size differences (Figure 4). The outer glume cell layer of groups D and J contained substantially more cells (16.5\% and $15.3 \%$, respectively) compared to that of Ilpumbyeo, with only a $1.3 \%$ and $1.4 \%$ increase in cell length, respectively. These data indicate that the increased width of group D and J spikelet hulls mainly results from an increase in cell number, rather than cell size, indicating that $q T G W 6$ may be involved in the regulation of cell division.

\section{Impact of the QTL cluster on yield per plant}

Eleven groups containing $44 \mathrm{~F}_{5}$ lines were used for the yield trials, together with Ilpumbyeo and IL28 in 2013 (Figure 3). IL28 had significantly higher grain yield compared to Ilpumbyeo. IL28 produced $8 \%$ higher grain yield on average compared to Ilpumbyeo. All groups with the Moroberekan segment at both qSPP6 and qTGW6 (i.e., groups D, E, F, G, H, and I) had significantly higher grain yield values compared with the 
a
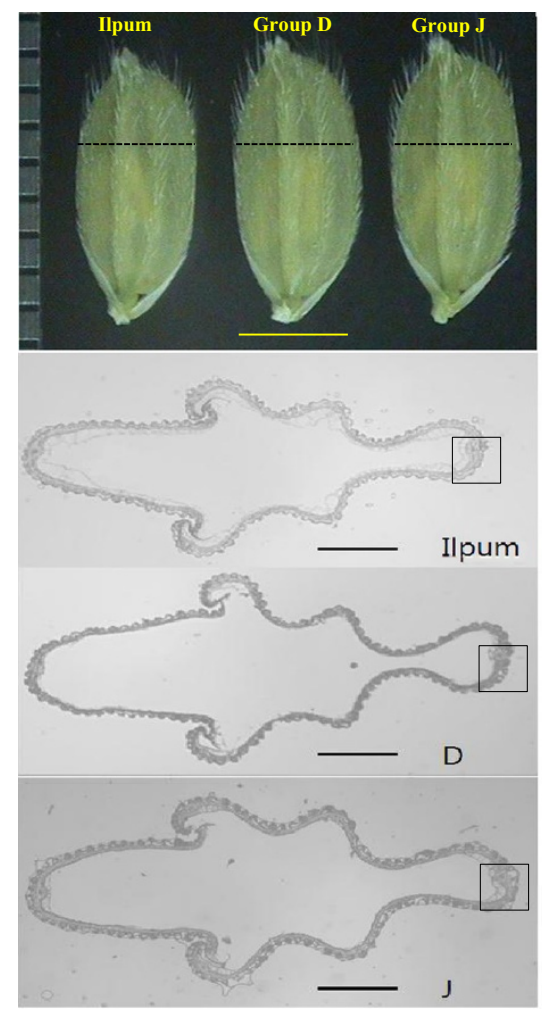

$\mathrm{b}$
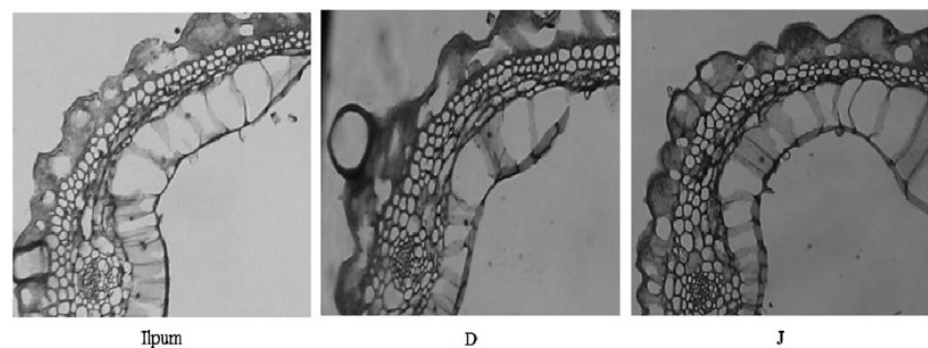

c

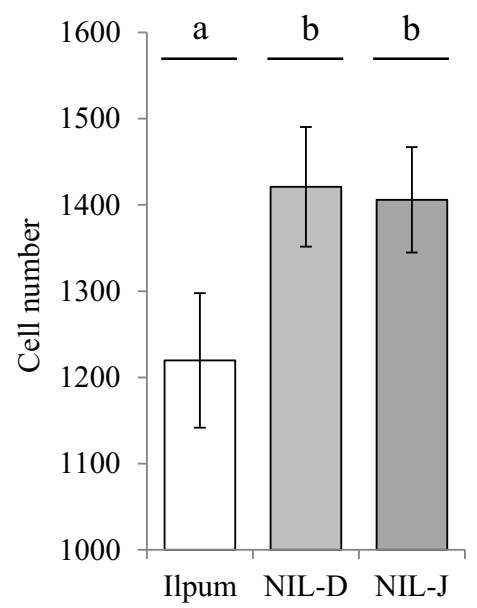

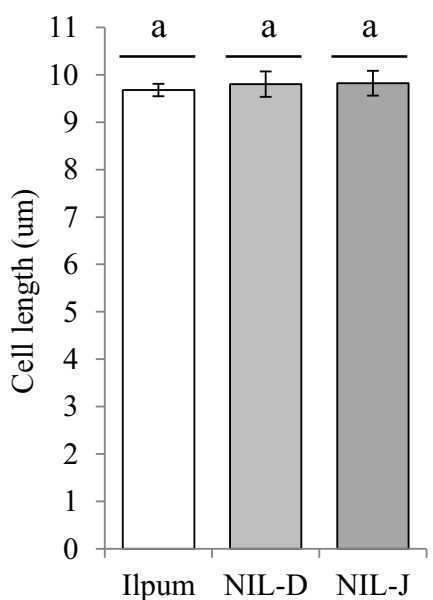

Figure 4 Histological analyses of spikelet hull 3 days before heading in Ilpumbyeo, group D, and group J. (a) Cross-section of spikelet hull. Upper: spikelets (scale bar, 3 mm). Low: cross-section of spikelet hull (scale bar, 500um). Dotted line indicates position of cross-section (b) Magnified view of spikelet hull cross-section boxed in a. (c) Comparison of total cell number and mean cell length in the outer glume cell layers of spikelet hull of Ilpumbyeo, group D, and group J. ( $n=5$ to 8 spikelets). Numbers followed by the same letter on each box are not significantly different at $P=0.05$ based on Duncan's multiple range test.

groups without the Moroberekan segment at both $q S P P 6$ and $q T G W 6$ (i.e., groups A, B, C, and K) and Ilpumbyeo (Figure 3). Also, the yield per plant of group J with qTGW6 and without qSPP6 was higher than that of group $C$ and Ilpumbyeo at $P<0.1(P=0.068, P=0.067$, respectively), and this result implies the contribution of qTGW6 to the yield increase. However, we could not determine the effect of $q S P P 6$ on yield due to the lack of line(s) only with the Moroberekan segment at qSPP6.

\section{Candidate genes for qTGW6}

qTGW6 was defined in a $37.85-\mathrm{kb}$ region between InDel-1 and SNP3. On the basis of available sequence annotation databases (http://www.gramene.org), there are four predicted genes in the target region. The functional annotations of the four genes are as follows: Loc_Os06g45550 (five exons and four introns) and Loc_Os06g45560 (eight exons and seven introns) are retrotransposon protein putative expressed genes; Loc_Os06g45570 (one exon) is a VQ domain-containing protein putative expressed gene; Loc_Os06g45580 (one exon) is a RING-H2 zinc finger putative protein expressed gene (Figure 3). We compared the genomic sequences of the open reading frame (ORF) and promoter region of the four genes between Ilpumbyeo and Moroberekan. Comparison of the genomic sequence of two genes (Loc_Os06g45550 and Loc_Os06g45560) among Nipponbare, Ilpumbyeo, and Moroberekan revealed the existence of a single non-synonymous SNP in the third and fourth exon of Loc_Os06g45550 and Loc_Os06g45560, respectively. The non-synonymous SNP at the third exon of Loc_Os06g45550 is characterized by the nucleotide Cytosine in Ilpumbyeo and the nucleotide Adenine in Moroberekan. The change in the nucleotide base from Cytosine to Adenine resulted in missense mutation from cysteine to tyrosine. The nonsynonymous SNP at the fourth exon of Loc_Os06g45560, is characterized by the nucleotide Adenine in Ilpumbyeo and the nucleotide Guanine in Moroberekan. The nucleotide substitution A (adenine) to $G$ (guanine) in Loc_Os06g45560 resulted in a missense mutation from Threonine to Alanine. No sequence variation in open reading frames and the promoter region of two genes was detected for Loc_Os06g45570 and Loc_Os06g45580 among the three varieties, Nipponbare, Ilpumbyeo, and Moroberekan. 


\section{Discussion}

There has been much debate as to whether similar genomic locations of QTLs that affect different traits are caused by the pleiotropy of a single gene or the tight linkage of several genes that individually influence specific traits. The question of pleiotropy versus tight linkage might be resolved by using a large-size population combined with high-density mapping, because two linked QTLs segregate independently in NIL- $\mathrm{F}_{2: 3}$ populations. Recently, several QTLs controlling the same or multiple traits have been confirmed to be closely linked in a small genomic region. For example, the rice photoperiod sensitivity gene, $H d 3$ was originally detected as a heading date-related quantitative trait locus that is localized on chromosome 6 in the $F_{2}$ population (Yano et al. 1997). For the fine-scale mapping of $H d 3$, highresolution linkage analysis using a large segregating population derived from advanced backcross progeny between the japonica rice variety, Nipponbare and the indica variety, Kasalath, was carried out. As a result of the high-resolution linkage mapping of $H d 3$, two tightly linked loci, $H d 3 a$ and $H d 3 b$, which promote flowering under short-day conditions and inhibit heading under long-day conditions, were identified in the $\mathrm{Hd} 3$ region (Monna et al. 2002). Lou et al. (2013) mapped two QTLs (qSPP5 and qTGW5) for spikelets per panicle and 1,000-grain weight to the same region on chromosome 5. Substitution mapping with the $\mathrm{BC}_{5} \mathrm{~F}_{3}$ and $\mathrm{BC}_{5} \mathrm{~F}_{4}$ populations derived from a cross between QTL-NIL and the recurrent parent, demonstrated that two tightly linked QTLs ( 9 SPP5 and $q T$ TWW5) were different. Several QTLs with large pleiotropic effects on multiple traits have also been found and cloned (APO1, Ghd7, and Ghd8 or $D T H 8)$. For instance, Hua et al. (2002) reported that a QTL cluster influences multiple traits (namely, grain per panicle, plant height, and heading date) on chromosome 7 in a primary population. High-resolution mapping with a large-sized segregating population and complementation tests revealed that these three traits are controlled by a single locus, designated as Ghd7 (Xue et al. 2008).

The original target of this study was $q S P P 6$, which was mapped on the long arm of chromosome 6 (Ju et al. 2008). In the process of the fine-scale mapping, the QTL for additional yield components (including panicle length, node width, and 1,000-grain weight) were consistently detected in the same region. A total of $243 \mathrm{~F}_{2}$ plants derived from a cross between NIL (IL28) and Ilpumbyeo for genetic analysis was not large enough to clarify the relationship between SPP and TGW. To confirm the precise location of the two QTLs, substitution mapping was carried out. Following substitution mapping with $44 \mathrm{~F}_{3: 4: 5}$ lines, $q S P P 6$ was mapped to a $431-\mathrm{kb}$ interval between RM20521 and SNP1, while qTGW6 was mapped to a $37.85-\mathrm{kb}$ interval between InDel-1 and SNP3, based on the japonica genome sequence. Therefore, the development of NIL and substitution mapping has enabled us to map SPP and TGW at the fine-scale.

No TGW-associated QTL was detected near the qTGW6 QTL in this study, indicating that qTGW6 is a novel QTL for TGW. Among the four predicted genes in the target region between InDel-1 and SNP-3 (37.85-kb), Loc_Os06g45570 encodes a VQ domain-containing protein. VQ domains are believed to be critical for the defense response to pathogens by interacting with WRKY transcription factors (Tao et al. 2011). Loc_Os06g45580 encodes the RING-H2 zinc finger protein. Recent studies reported a critical role of the ubiquitin pathway in grain development in rice (Song et al. 2007; Weng et al. 2008). $G W 2$, a major QTL for rice grain width and weight encodes a new RING-type E3 ubiquitin ligase. GW5, another major QTL underlying rice width and weight, is likely to act in the ubiquitine-proteasome pathway to regulate cell division during cell division (Weng et al. 2008). qTGW6 exhibited common function for grain width with GW2 and GW5 in that it was associated with increased cell numbers, a wider spikelet hull and an increased grain milkfilling rate. This raises a possibility that Loc_Os06g45580 is a candidate for $q T G W 6$. However, no sequence variation in open reading frames and the promoter regions of two genes, Loc_Os06g45570 and Loc_Os06g45580 was observed between Ilpumbyeo and Moroberekan and this result seems to suggest that they are not candidate genes for qTGW6. The other two genes in the target region are retrotransposon proteins, Loc_Os06g45550 and Loc_Os06g45560. Loc_Os06g45560 was transcribed in seedling and embryo/endosperm 25 days after pollination but Loc_Os06g45550 was not transcribed based on the available gene expression database (RGAP, http://rice.plantbiology.msu.edu/expression.shtml).

More than $40 \%$ of rice genome is composed of repetitive sequences and transposable elements (Goff et al. 2002). A recent study showed that the expression of Tos17, a copia-like retrotransposon was affected by overexpression or down-regulation of several rice SUVH (Su(var) 3-9 homologs) genes and RNAi plants with downregulated SDG728, a rice SUVH gene showed a reduced seed size phenotype (Qin et al. 2010). This raises a possibility that a relationship between altered seed morphology and overexpression of Tos17 might exist. Interestingly, our alignment analysis of GW5 a.a sequence using BLASTP program showed that GW5 protein has high homology with two retrotransposon proteins encoded by LOC_Os08g26160.1 and LOC_Os05g47720.1 (data not shown), although it has been reported that the protein encoded by the GW5 ORF had no sequence homology with any proteins of known molecular function (Weng et al., 2008). GW5 
protein showed 56\% homology to the retrotransposon protein with 194 a.a encoded by LOC_Os08g26160.1 and $47-50 \%$ homologies to the transposon protein with 411 a.a. by LOC_Os05g47720.1. Numerous Indels and single-nucleotide polymorphisms (SNPs) were detected between Nipponbare (japonica) and 9311 (indica) and Indels are mainly caused by transposons (Feng et al. 2002). Indels and SNPs in some key domestication genes including $q S H 1$ and $G W 2$ are responsible for morphological changes occurred during domestication (Konishi et al. 2006; Song et al. 2007). Also, the difference in grain width between japonica and indica cultivars is associated with a 1212-bp deletion harboring the GW5 ORF which was possibly caused by transposons (Weng et al. 2008). These observations support our hypothesis that the protein encoded by the GW5 ORF might be a retrotransposon and Loc_Os06g45560 is a candidate for $q T G W 6$. However, the presence of SNPs in the upstream regions of the genes between two parents suggests the possibility that a long-distance regulation is involved in controlling the qTGW6 (data not shown). Konishi et al. (2006) reported that the casual mutation in $\mathrm{qSH} 1$ underlying the seed shattering was found in the 12-kb upstream region from the ORF of the gene and caused the absence of the abscission layer in Nipponbare. Additional fine mapping experiments are underway to determine the functional relationship between qTGW6 and GW5 in controlling seed development. Also, loss-of-function mutant lines of Loc_Os06g45560 will be evaluated for grain morphology traits and the gene expression. These informations on the molecular mechanism of $q T G W 6$ would be necessary to get more insights into the mechanism of grain development.

Although the candidate gene of $q T G W 6$ and its genetic mechanism was not elucidated here, we found that the Moroberekan chromosomal segment at this region (a tropical japonica) harbors QTLs for yield component traits leading to an yield increase in japonica cultivar. Yield trials confirmed that introgression lines containing the Moroberekan segment at the qSPP6 and qTGW6 loci produced significantly higher yields than Ilpumbyeo $(P<0.05)$. The grain yield per plant in IL28 was $8 \%$ higher than that of Ilpumbyeo. Moroberekan alleles in the target region on chromosome 6 had a favorable effect on yield, by increasing the number of spikelets per panicle and 1,000-grain weight, in addition to lodging tolerance by increasing the node width, whereas it had no negative effect on heading date and plant height. Thus, the qSPP6 and $q T G W 6$ alleles are potentially valuable for improving rice yield. It is proposed that NILs containing the target segment associated with positive QTLs from Moreberekan should be developed from this population, and evaluated in a wide range of environments, to assess the interaction between QTLs and the environment. Tightly linked SSR markers are expected to facilitate the cloning of genes underlying these QTLs, in addition to marker-assisted selection for variation in the SPP and TGW in rice breeding programs.

\section{Conclusion}

In this study, we demonstrated that two QTLs, qSPP6 for spikelets per panicle (SPP) and qTGW6 for 1,000grain weight (TGW) are tightly linked on chromosome 6. Because Moroberekan alleles for the SPP and TGW have been shown to be beneficial in the genetic background of Ilpumbyeo, the qSPP6 and qTGW6 alleles may prove valuable for improving the yield potential of japonica rice cultivars. Tightly linked SSR markers are expected to facilitate the cloning of genes underlying these QTLs, in addition to marker-assisted selection for variation in SPP and TGW in rice breeding programs.

\section{Methods}

\section{Plant materials}

In a previous study (Kim et al. 2013), IL28 was shown to exhibit a higher number of spikelets per panicle and heavier grain compared to the recurrent parent, and contained the Moroberekan chromosomal segment at the $q S P P 6$ region on chromosome 6; hence, it was selected for the fine-scale mapping of qSPP6 in the current study. IL28 was crossed with the cultivar Ilpumbyeo, with the resulting $F_{1}$ plants being self-pollinated to obtain an $F_{2}$ population (234 plants in 2009 and 1,150 plants in 2010). To confirm qSPP6, $44 \mathrm{~F}_{2}$ homozygous plants with different cross-over breakpoints between RM7269 and RM20653 were selected and selfed to produce $\mathrm{F}_{3}$ lines for the phenotyping and substitution mapping of $q S P P 6$. In the process of the fine-scale mapping of qSPP6, a QTL for 1,000-grain weight (TGW) was consistently detected in the same region. To confirm the precise location of $q S P P 6$ and $q T G W 6$, these $44 \mathrm{~F}_{3}$ lines were advanced to $F_{4}$ and $F_{5}$ lines, which were used to evaluate spikelets per panicle, grain weight, grain morphology traits, and grain yield.

\section{Field trial and trait evaluation}

A total of $243 \mathrm{~F}_{2}$ plants and $1150 \mathrm{~F}_{2}$ plants (2009 and 2010), and $41 \mathrm{~F}_{3: 4: 5}$ lines (2011, 2012, and 2013) and the parental lines were grown at the experimental field of Chungnam National University, Daejeon, Korea. In 2009 , the 243 plants and the parents were planted at distances of $30 \times 15 \mathrm{~cm}$, and evaluated for grain (brown rice) weight (150 out of 243 plants were used to evaluate grain weight). In 2010, for the substitution mapping of qTGW6, 1,150 $\mathrm{F}_{2}$ plants were genotyped with four additional markers being located between RM7269 and RM20653 (RM20512, RM20521, RM20652, and RM20572) and one marker (RM551) located on chromosome 4. The 
experiment using $44 \mathrm{~F}_{3: 4: 5}$ lines derived from $44 \mathrm{~F}_{2}$ plants followed a completely randomized block design with two replications, one row per plot, and 25 plants per row in 2011, 2012, and 2013. The middle 10 plants from each line were chosen for the evaluation, and the average of the measurements was used for the phenotype of each line for the spikelets per panicle (SPP) and 1,000-grain weight (TGW). SPP were measured by averaging the three major panicles per plants. Grains with hulls were allowed to dry naturally after harvesting, and partial or unfilled seeds were removed by soaking grains in water. Fully filled seeds were re-dried in an oven at $30^{\circ} \mathrm{C}$ for $24 \mathrm{~h}$. The TGW was evaluated by measuring the weight of 250 randomly selected, de-hulled grains per plant (10 plants per line). In 2013, grain length $(\mathrm{GL})$, grain width $(\mathrm{GW})$, grain thickness $(\mathrm{GT})$, and yield per plants (YD) were measured. The grain length (GL), grain width $(\mathrm{GW})$, and grain thickness (GT) were measured for 50 grains (brown rice) per plant (10 plants per line) using a 150-mm Vernier caliper (Mitutoyo Corp., Japan). Yield per plant (YD) was measured by averaging the grain yield (g) of 10 plants that were randomly selected from the center of each plot per block. The 1,000-grain weight and the yield per plant were corrected for the $12 \%$ grain moisture content.

\section{DNA extraction and SSR analysis}

DNA was extracted from the leaf tissue of the $F_{2}$ population using a chloroform-based DNA extraction protocol (Causse et al., 1994). A 20- $\mu \mathrm{L}$ reaction mixture was used, containing $5.0 \mu \mathrm{L}(5 \mathrm{ng} / \mu \mathrm{L})$ of template DNA, $0.1 \mu \mathrm{l}$ (5 Unit/ $\mu \mathrm{L}$ ) Taq polymerase, $0.8 \mu \mathrm{l}$ dNTP (2.5 mM each), Forward + Reverse primer $1 \mu \mathrm{l}(10 \mathrm{pmol}$ each), $2.0 \mu \mathrm{l} 10 \times$ PCR buffer (10 mM Tris- $\mathrm{HCl} \mathrm{PH} 8.3,50 \mathrm{mM} \mathrm{KCl}$, $1.5 \mathrm{mM} \mathrm{MgCl} 2,0.1 \%$ gelatin), and $11.1 \mu \mathrm{l}$ triple-distilled water. Amplification was achieved using a Thermo Cycler (Bio-Rad) based on the step-cycle program set for denaturation at $94^{\circ} \mathrm{C}$ for $5 \mathrm{~min}$, subsequent denaturation was performed at $94^{\circ} \mathrm{C}$ for $1 \mathrm{~min}$, annealing at $55^{\circ} \mathrm{C}$ for $1 \mathrm{~min}$, extension at $72^{\circ} \mathrm{C}$ for $1 \mathrm{~min}$; steps 2 to 4 were repeated for a total of 35 cycles, with a final extension step at $72^{\circ} \mathrm{C}$ for $5 \mathrm{~min}$. PCR products were run on $4 \%$ polyacrylamide denaturing gels for 1-2 h at 1800-2000 V, and marker bands were revealed by silver staining (Panaud et al. 1996). The orientation of the SSR markers was based on the SSR maps (McCouch et al. 2002). Due to the lack of markers showing polymorphism in the region between RM20562 and
RM20572, additional genotyping of the $F_{3}$ lines was conducted with four targeted SNPs and one Insertion/Delition marker. To detect the SNPs and InDels of Ilpumbyeo and Moroberekan, resequencing with NGS (Next-Generation Sequencing) was conducted using the two parents according to Jeong et al. (2013). Through the comparison of genomic sequences corresponding to the region between RM20562 and RM20572, a total of 28 polymorphisms (14 SNPs, and 14 Insertion/Deletions) were detected between Ilpumbyeo and Moroberekan. The polymorphisms were assayed by the direct sequencing of the four regions. Information on the primers used is presented in Table 2 . The primers were designed according to the Nipponbare sequence (http://rgp. dna.affrc.go.jp/E/IRGSP/Build5/build5.html). The first InDel, hereafter referred to as InDel-1, occurs at the 27,565,774th position based on the Nipponbare sequence (www.gramene. org), and is characterized by nucleotide - (1 bp deletion) in Ilpumbyeo and nucleotide A in Moroberekan. The first SNP, SNP-1, that occurs at the 27,572,767th position, is characterized by nucleotide $\mathrm{G}$ in Ilpumbyeo and nucleotide $\mathrm{A}$ in Moroberekan. The second SNP, SNP-2, that occurs at the $27,586,202$ th position, is characterized by nucleotide $\mathrm{A}$ in Ilpumbyeo and nucleotide $\mathrm{G}$ in Moroberekan. The third SNP, SNP-3, that occurs at the 27,629,080th position, is characterized by nucleotide $\mathrm{A}$ in Ilpumbyeo and nucleotide $\mathrm{C}$ in Moroberekan.

\section{Statistical analysis}

Statistical analysis was performed using Qgene software version 2.30 for Macintosh (Nelson 1997) and SAS (SAS Institute). Single point analysis (SPA) was performed to determine the effect of each marker on each trait. In SPA, a QTL was confirmed if the phenotype was associated with a marker locus at $P<0.005$, or with two adjacent marker loci at $P<0.01$. The proportion of total phenotypic variation explained by each QTL was calculated as an $\mathrm{R}^{2}$ value, from the regression of each marker/ phenotype combination. QTLs were mapped at a finescale by comparing the phenotypic means of two genotypic classes of recombinants (Ilpumbyeo homozygote and Moroberekan homozygote) within the target region by using the SAS statistical software package.

\section{Histological analysis}

Paraffin-embedded sections of spikelet samples were prepared according to $\mathrm{Li}$ et al. (2009), with minor

Table 2 Primer sequences of newly developed SNP markers

\begin{tabular}{lllll}
\hline Marker name & Forward primer $\left.\mathbf{( 5}^{\prime} \mathbf{-} \mathbf{3}^{\prime}\right)$ & Reverse primer $\left(\mathbf{5}^{\prime} \mathbf{-} \mathbf{3}^{\prime} \mathbf{)}\right.$ & Position & Product size (bp) \\
\hline InDel-1 & GAGAAAGTGACCTCGTTAGT & CAAGACATGACACTACGATTGC & $27,565,623-27,565,899$ & 276 \\
SNP1 & CCATATGATTAAATGGGAGGCTC & ACATAGGCACTGCCAAGGTC & $27,572,630-27,572,896$ & 266 \\
SNP2 & ACGGACGCCTGCTATAGGGA & CACCAGAGGCGATCTTCTTC & $27,585,994-27,586,360$ & 366 \\
SNP3 & GAATATCACGATCAAAGAACTTG & GTTACTCTAATATGATAATATGCC & $27,603,470-27,603,822$ & 352 \\
\hline
\end{tabular}


modifications. Plant materials were fixed in FAA $(50 \%$ ethanol, 5\% glacial acetic acid and 5\% formaldehyde) and stored at $4^{\circ} \mathrm{C}$ for $24 \mathrm{~h}$. The fixed spikelets were dehydrated by soaking them for $6 \mathrm{~h}$ in a gradient ethanol series $(60 \%, 70 \%, 80 \%, 90 \%)$, and were then incubated in $100 \%$ ethanol overnight. Dehydrated spikelets were embedded in Paraplast (Sigma). Tissue sections ( $8 \mu \mathrm{m}$ thick) were cut using a rotary microtome, transferred onto gelatin-coated glass slides, and dried at $42^{\circ} \mathrm{C}$ for 1 day. The sections were de-paraffinized with 100\% xylene for $5 \mathrm{~min}$, followed by soaking for $2 \mathrm{~min}$ in $50 \%$ ethanol $/ 50 \%$ xylen, $100 \%$ ethanol, and sterile water. The samples were stained with toluidine blue for $30 \mathrm{~s}$, and washed twice with sterile water. The samples were then soaked for 2 min in 70\% ethanol, 80\% ethanol, 90\% ethanol, and $100 \%$ ethanol. Finally, the samples were cleaned by soaking them twice in $100 \%$ xylen. The sections were photographed under an Olympus BH2-RFCA using a U-PMTVC camera.

\section{Competing interests}

The authors declare that they have no competing interests.

\section{Authors' contributions}

DK participated in phenotyping, genotyping, drafting the manuscript. $H L, M F$ and JK participated in phenotyping. SK participated in sequencing, primer design and genotyping. SA conceived of the study, drafted proposal and corrected manuscript. All authors have read and approved the manuscript.

\section{Acknowledgements}

This work was supported by the Korea Research Foundation Grant funded by the Korean Government (MOEHRD, Basic Research Promotion Fund) (KRF-2010-0024118) and the Next-Generation Biogreen 21 Program (Plant Molecular Breeding Center No. PJ008136), Rural Development Administration, Republic of Korea.

\section{Author details}

${ }^{1}$ Department of Agronomy, College of Agriculture \& Life Sciences, Chungnam National University, Daejeon 305-764, South Korea. ${ }^{2}$ Nationa Academy of Agricultural Science, Rural Development Administration, Suweon 441-707, South Korea. ${ }^{3}$ Chungnam Agricultural Research and Extension Services, Yesan 340-861, South Korea. ${ }^{4}$ Present address: Department of Variety Testing, Korea Seed \& Variety Service, Ministry of Agriculture, Food and Rural Affairs, Kimcheon 740-220, South Korea.

Received: 2 January 2014 Accepted: 14 July 2014

Published online: 19 August 2014

\section{References}

Ando T, Yamamoto T, Shimizu T, Ma XF, Shomura A, Takeuchi Y, Lin SY, Yano M (2008) Genetic dissection and pyramiding of quantitative traits for panicle architecture by using chromosomal segment substitution lines in rice. Theor Appl Genet 116:881-890

Ashikari M, Sakakibara H, Lin SY, Yamamoto T, Takashi T, Nishimura A, Angeles ER, Qian Q, Kitano H, Matsuoka M (2005) Cytokinin oxidase regulates rice grain production. Science 309:741-745

Causse MA, Fulton TM, Cho YG, Ahn SN, Chunwonse J, Wu K, Xiao J, Yu Z, Ronald PC, Harrington SE, Second G, McCouch SR, Tanksley SD (1994) Saturated molecular map of the rice genome based on an interspecific backcross population. Genetics 138:1251-1274

Cui KH, Peng SB, Xing YZ, Yu SB, Xu CG, Zhang Q (2003) Molecular dissection of the genetic relationships of source-sink and transport tissue with yield traits in rice. Theor Appl Genet 106:649-658

Feng Q, Zhang Y, Hao P, Wang S, Fu G, Huang Y, Li Y, Zhu J, Liu Y, Hi X, Jia P, Zhang Y, Zhao Q, Ying K, Yu S, Tang Y, Weng Q, Zhang L, Lu Y, Mu J, Lu Y,
Zhang LS, Yu Z, Fan D, Liu X, Lu T, Li C, Wu Y, Sun T, Lei H et al (2002) Sequence and analysis of rice chromosome 4. Nature 420:316-320

Goff SA, Ricke D, Lan TH, Presting G, Wang R, Dunn M, Glazebrook J, Sessions A, Oeller P, Varma H, Hadley D, Hutchison D, Martin C, Katagiri F, Lange BM, Moughamer T, Xia Y, Budworth P, Zhong J, Miguel T, Paszkowski U, Zhang S, Colbert M, Sun WL, Chen L, Cooper B, Park S, Wood TC, Mao L, Quail P, Wing $R$, Dean $R$ et al (2002) A draft sequence of the rice genome (Oryza sativa $L$. ssp. japonica). Science 296:92-100

Hua JP, Xing YZ, Xu CG, Sun XL, Yu SB, Zhang Q (2002) Genetic dissection of an elite rice hybrid reveal that heterozygotes are not always advantageous for performance. Genetics 162:1885-1895

Huang X, Qian Q, Liu Z, Sun H, He S, Luo D, Xia G, Chu C, Li J, Fu X (2009) Natural variation at the DEP1 locus enhances grain yield in rice. Nat Genet 41:494-497

Ikeda K, Ito M, Nagasawa N, Kyozuka J, Nagato Y (2007) Rice ABERRANT PANICLE ORGANIZATION 1, encoding an F-box protein, regulates meristem fate. Plant J 51:1030-1040

Ishimaru K (2003) Identification of a locus increasing rice yield and physiological analysis of its function. Plant Physiol 133:1083-1090

Ishimaru K, Hirotsu N, Madoka Y, Murakami N, Hara N, Onodera H, Kashiwagi T, Kazuhiro U, Shimizu B, Onishi A, Miyagawa H, Katoh E (2013) Loss of function of the IAA-glucose hydrolase gene TGW6 enhances rice grain weight and increases yield. Nat Genet 45:707-711

Jeong IS, Yoon UH, Lee GS, Ji HS, Lee HJ, Han CD, Hahn JH, An GH, Kim TH (2013) SNP-based analysis of genetic diversity in anther-derived rice by whole genome sequencing. Rice 6:6

Ji SD, Luo XL, Ahn SN (2014) Mapping QTL for ratooning ability in advanced backcross lines from an Oryza sativa $\times$ O. rufipogon cross. CNU J Agricultural Sci 41(1):1-7

Jin F, Kim DM, Ju HG, Ahn SN (2009) Mapping quantitative trait loci for awnness and yield component tratis in isogenic lines derived from an Oryza sativa/O rufipogon cross. J Crop Sci Biotech 12(1):9-16

Ju HG, Kim DM, Kang JW, Kim MK, Kim YG, Ahn SN (2008) Mapping QTLs for agronomic traits using an introgression line population from a cross between Ilpumbyeo and Moroberekan in rice. Korean J Breed 40(4):414-421

Kim DM, Kim JH, Kang JW, Lee HS, Ahn SN (2013) Fine mapping of a quantitative trait loci controlling the number of spikelets per panicle in rice. Korean $J$ Breed 44(4):462-469

Khush GS (1999) Green revolution: preparing for the 21st century. Genome 42:646-655

Khush GS (2005) What it will take to feed 5.0 billion rice consumers in 2030. Plant Mol Biol 59:1-6

Konishi S, Izawa T, Lin S, Ebana K, Fukuta Y, Sasaki T, Yano M (2006) An SNP caused loss of seed shattering during rice domestication. Science 312:1392-1396

Li C, Zhou A, Sang T (2006) Gentetic analysis of rice domestication syndrome with the wild annual species. Oryza Nivara New Phytol 170:185-194

Li H, Wue D, Gao Z, Yan M, Xu W, Xing Z, Huang D, Qian Q, Wue Y (2009) A putative lipase gene EXTRA GLUME1 regulates both empty-glume fate and spikelet development in rice. Plant J 57:593-605

Lin HX, Qian HR, Zhuang JY, Lu J, Min SK, Xiong ZM, Huang N, Zheng KL (1996) RFLP mapping of QTLs for yield and related characters in rice (Oryza sativa L.). Theor Appl Genet 92:920-927

Linh HL, Hang NT, Jin FX, Kang KH, Lee YT, Kwon KH, Ahn SN (2008) Introgression of a quantitative trait locus for spikelets per panicle from Oryza minuta to the O. sativa cultivar Hwaseongbyeo. Plant Breed 127:262-267

Lou X, Ji SD, Yuan PR, Lee HS, Kim DM, Balkunde S, Kang JW, Ahn SN (2013) QTL mapping reveals a tight linkage between QTLs for grain weight and panicle spikelet number in rice. Rice 6:33

McCouch SR, Teytelman L, Xu Y, Lobos KB, Clare K, Walton M, Fu B, Maghirang R, Li Z, Xing Y, Zhang Q, Kono I, Yano M, Fjellstrom R, DeClerck G, Schneider D, Cartinhour S, Ware D, Stein D (2002) Development and mapping of 2240 new SSR markers for rice (Oryza sativa L.). DNA Res 9:199-207

Miura K, Ikeda M, Matsubara A, Song XJ, Ito M, Asano K, Matsuoka M, Kitano H, Ashikari M (2010) OsSPL14 promotes panicle branching and higher grain productivity in rice. Nat Genet 42:545-549

Monna L, Lin X, Kojima S, Sasaki T, Yano M (2002) Genetic dissection of a genomic region for a quantitative trait locus, $\mathrm{Hd} 3$, into two loci, $\mathrm{Hd} 3 \mathrm{a}$ and Hd3b, controlling heading date in rice. Theor Appl Genet 104:772-778

Nelson JC (1997) QGENE: software for marker-based genomic analysis and breeding. Mol Breed 3:239-245 

M, Nishitani R, Ebitani T, Ozaki H, Angeles ER, Hirasawa T, Matsuoka M (2010) New approach for rice improvement using a pleiotropic QTL gene for lodging resistance and yield. Nat Comms 1-132, doi:10. 1038/ncomms1132

Panaud O, Chen X, McCouch SR (1996) Development of microsatellite markers and characterization of simple sequence length polymorphism (SSLP) in rice (Oryza sativa L.). Mol Gen Genet 252:597-607

Qin FJ, Sun QW, Huang LM, Chen XS, Zhou DX (2010) Rice SUVH histone methyltransferase genes display specific functions in chromatin modification and retrotransposon repression. Mol Plant 3(4):773-782

Song XJ, Huang W, Shi M, Zhu MZ, Lin HX (2007) A QTL for rice grain width and weight encodes a previously unknown RING-type E3 ubiquitin ligase. Nat Genet 39:623-630

Suh JP, Ahn SN, Cho YC, Suh HS, Hwang HG (2005) Mapping of QTLs for yield traits using an advanced backcross population from a cross between Oryza sativa and O. glaberrima. Korean J Breed 37(4):214-220

Tao Z, Kou Y, Liu H, Li X, Xiao J, Wang S (2011) OsWRKY45 alleles play different roles in abscisic acid signaling and salt stress tolerance but similar roles in drought and cold tolerance in rice. J Exp Bot 62:4863-4874

Thomson MJ, Tai T, McClung AM, Lai XH, Hinga ME, Lobos KB, Xu Y, Martinez CP, McCouch SR (2003) Mapping quantitative trait loci for yield components and morphological traits in an advanced backcross population between Oryza rufipogon and the Oryza sativa cultivar Jefferson. Theor Appl Genet 107:479-493

Weng J, Gu S, Wan X, Gao H, Guo T, Su N, Lei C, Zhang X, Cheng Z, Guo X, Wang J, Zhai H, Wan J (2008) Isolation and initial characterization of GW5, a major QTL associated with rice grain width and weight. Cell Res 18:1199-1209

Xie X, Jin F, Song MH, Suh JP, Hwan HG, Kim YG, McCouch SR, Anh SN (2008) Fine mapping of a yield-enhancing QTL cluster associated with transgressive variation in an Oryza sativa X O. rufipogon cross. Theor Appl Genet 116:613-622

Xiong LZ, Liu KD, Dai XK, Xu CG, Zhang Q (1999) Identification of genetic factors controlling domestication related traits of rice using an $\mathrm{F}_{2}$ population of a cross between Oryza sativa and O. rufipogon. Theor Appl Genet 98:243-251

Xue W, Xing Y, Weng X, Zhao Y, Tang W, Wang L, Zhou H, Yu S, Xu C, Li X, Zhang Q (2008) Natural variation in Ghd7 is an important regulator of heading date and yield potential in rice. Nat Genet 40:761-767

Yamagishi M, Takeuchi Y, Kono I, Yano M (2002) QTL analysis for panicle characteristics in temperate japonica rice. Euphytica 128:219-224

Yano M, Harushima Y, Nagamura Y, Kurata N, Minobe Y, Sasaki T (1997) Identification of quantitative trait loci controlling heading date in rice using a high-density linkage map. Theor Appl Genet 95:1025-1032

Zhang Q (2007) Strategies for developing green super rice. Proc Natl Acad Sci U S A 104:16402-16409

Zhang Y, Luo L, Liu T, Xu C, Xing Y (2009) Four rice QTLs controlling number of spikelets per panicle expressed the characteristics of single Mendelian gene in near isogenic backgrounds. Theor Appl Genet 118:1035-1044

Zhuang JY, Lin HX, Lu J, Qian HR, Hittalmani S, Huang N, Zheng KL (1997) Analysis of QTL X environment interaction for yield components and plant height in rice. Theor Appl Genet 95:799-808

doi:10.1186/s12284-014-0014-5

Cite this article as: Kim et al:: High-density mapping of quantitative trait loci for grain-weight and spikelet number in rice. Rice 2014 7:14.

\section{Submit your manuscript to a SpringerOpen ${ }^{\circ}$ journal and benefit from:}

- Convenient online submission

$\checkmark$ Rigorous peer review

- Immediate publication on acceptance

- Open access: articles freely available online

- High visibility within the field

- Retaining the copyright to your article

Submit your next manuscript at $\boldsymbol{~ s p r i n g e r o p e n . c o m ~}$ 